\title{
Genome-wide analysis of long non-coding RNAs (IncRNAs) in two contrasting soybean genotypes subjected to phosphate starvation
}

Jinyu Zhang ${ }^{1 \dagger}$, Huanqing $\mathrm{Xu}^{2 \dagger}$, Yuming Yang ${ }^{2}$, Xiangqian Zhang ${ }^{2}$, Zhongwen Huang ${ }^{1 *}$ and Dan Zhang ${ }^{2^{*}}$ (1)

\begin{abstract}
Background: Phosphorus (P) is essential for plant growth and development, and low-phosphorus (LP) stress is a major factor limiting the growth and yield of soybean. Long noncoding RNAs (IncRNAs) have recently been reported to be key regulators in the responses of plants to stress conditions, but the mechanism through which LP stress mediates the biogenesis of IncRNAs in soybean remains unclear.

Results: In this study, to explore the response mechanisms of IncRNAs to LP stress, we used the roots of two representative soybean genotypes that present opposite responses to P deficiency, namely, a P-sensitive genotype (Bogao) and a P-tolerant genotype (NN94156), for the construction of RNA sequencing (RNA-seq) libraries. In total, 4,166 novel IncRNAs, including 525 differentially expressed (DE) IncRNAs, were identified from the two genotypes at different P levels. GO and KEGG analyses indicated that numerous DE IncRNAs might be involved in diverse biological processes related to phosphate, such as lipid metabolic processes, catalytic activity, cell membrane formation, signal transduction, and nitrogen fixation. Moreover, IncRNA-mRNA-miRNA and IncRNA-mRNA networks were constructed, and the results identified several promising IncRNAs that might be highly valuable for further analysis of the mechanism underlying the response of soybean to LP stress.

Conclusions: These results revealed that LP stress can significantly alter the genome-wide profiles of IncRNAs, particularly those of the P-sensitive genotype Bogao. Our findings increase the understanding of and provide new insights into the function of IncRNAs in the responses of soybean to P stress.
\end{abstract}

Keywords: LncRNAs, Phosphate starvation, RNA-Seq, Soybean

\footnotetext{
* Correspondence: hzw@hist.edu.cn; zhangd@henau.edu.cn

${ }^{\dagger}$ Jinyu Zhang and Huanqing Xu contributed equally to this work.

'School of Life Science and Technology, Henan Collaborative Innovation

Center of Modern Biological Breeding, Henan Institute of Science and

Technology, Xinxiang 453003, China

${ }^{2}$ Collaborative Innovation Center of Henan Grain Crops, College of

Agronomy, Henan Agricultural University, Zhengzhou 450002, China
}

(c) The Author(s). 2021 Open Access This article is licensed under a Creative Commons Attribution 4.0 International License, which permits use, sharing, adaptation, distribution and reproduction in any medium or format, as long as you give appropriate credit to the original author(s) and the source, provide a link to the Creative Commons licence, and indicate if changes were made. The images or other third party material in this article are included in the article's Creative Commons licence, unless indicated otherwise in a credit line to the material. If material is not included in the article's Creative Commons licence and your intended use is not permitted by statutory regulation or exceeds the permitted use, you will need to obtain permission directly from the copyright holder. To view a copy of this licence, visit http://creativecommons.org/licenses/by/4.0/ The Creative Commons Public Domain Dedication waiver (http://creativecommons.org/publicdomain/zero/1.0/) applies to the data made available in this article, unless otherwise stated in a credit line to the data. 


\section{Background}

In general, long noncoding RNAs (lncRNAs) refer to transcripts longer than 200 nucleotides and do not encode open reading frames (ORFs) [1]. In eukaryotes, most lncRNAs are transcribed by RNA polymerase II and have a structure similar to that of mRNA, which includes 5' capping, splicing and polyadenylation [2]. A growing body of evidence shows that lncRNAs play important functional roles in diverse biological processes, such as epigenetic regulation, cell cycle regulation, cellular growth and differentiation, by regulating the level of target genes $[3,4]$. LncRNAs are involved in a wide range of regulatory mechanisms that impact gene expression, including chromatin remodeling, modulation of alternative splicing, fine-tuning of miRNA activity, and the control of mRNA translation or accumulation [5].

Recent advances in biological technologies, such as tiling arrays and RNA deep sequencing (RNA-seq), have made it possible to survey the transcriptomes of many organisms to an unprecedented degree [6]. LncRNAs have been widely identified in various plants, such as Arabidopsis thaliana [7, 8], rice [9], Zea mays [10] and cotton [11]. Emerging studies have revealed that lncRNAs play important roles in various biological processes, including flowering regulation [12], photomorphogenesis [13], stress responses [14, 15] and other important developmental pathways [16, 17]. For example, the rice-specific lncRNA LDMAR has been identified as a key gene in controlling photoperiod-sensitive male sterility [18].

Plants possess an elaborate physiological system that responds to external abiotic stress conditions [19], including phosphorus $(\mathrm{P})$ deficiency. As one of the major mineral macronutrients present in all living things, $\mathrm{P}$ is essential for plant growth and development due to its key role in the regulation of energy metabolism and the synthesis of nucleic acids and membranes [20, 21]. Although $\mathrm{P}$ is abundant in soil, its direct use by plants is often limited due to its low bioavailability. Thus, low phosphorus (LP) stress represents a major limiting factor affecting plant growth and productivity [22]. P is important for plant growth and the agricultural industry, but it has been estimated that the $\mathrm{P}$ rock reserves will be depleted by 2050 [23]. Therefore, we need to understand the molecular mechanism underlying the responses of crops to LP stress and improve their phosphorus use efficiency. Plants have evolved numerous adaptive developmental and metabolic responses to cope with growth under phosphate-limited conditions, and these responses include modifying the root system architecture (RSA), increasing acid phosphatase activity (APA), and the release of low-molecular-weight organic acids [20]. Many studies have shown that many P-related genes, such as GmACP1 [22], GmHAD1 [24], and PHR1 [25], are involved in plant growth and development. Noncoding
RNAs serve as one of the key regulators involved in the $\mathrm{P}$ starvation response network. Changes in miRNAs, such as miR399 [26] and miR827 [27], constitute an important mechanism used by plants to adapt to LP environments. LncRNAs also play key roles in regulating the mRNA and/or miRNA levels of a large number of genes associated with P starvation responses [14, 28, 29], which suggests their important functions in regulating the responses of plants to LP stress. Du et al. found that PILNCR1 (long-noncoding RNA1) can inhibit the ZmmiR399-guided cleavage of $\mathrm{ZmPHO}$, and the interaction between PILNCR1 and miR399 is important for the tolerance of maize to LP conditions [28].

Soybean is not only a major crop plant constituting a major agricultural industry worldwide but also an important seed crop because it is an essential source of proteins, oils and micronutrients for human and livestock consumption [30]. Because soybean seeds contain higher concentrations of $\mathrm{P}$ than rice, wheat and corn, soybean requires more $\mathrm{P}$ than other crops to maintain its growth and development [31]. Previous studies have provided an understanding of the protein-coding genes and miRNAs involved in the response of soybean to phosphate starvation $[14,28,29]$, but the role of lncRNAs in the response of soybean to LP stress has rarely been reported.

In this study, two contrasting genotypes of soybean, namely, Bogao (a LP-sensitive genotype) and Nannong 94156 (a LP-tolerant genotype), were used to investigate the regulatory mechanism of lncRNAs under P starvation. Using genome-wide high-throughput RNA sequencing (RNA-seq) technology, we identified and characterized a total of 4,166 lncRNAs that are responsive to LP stress in the roots of soybean seedlings, validated 14 lncRNAs by qPCR, and identified 525 differentially expressed (DE) lncRNAs related to the regulation of the tolerance of soybean to LP stress. We then performed GO and KEGG analyses and constructed an LP-responsive network to explore the putative functions of the identified lncRNAs. The results lay the foundation for obtaining a more in-depth understanding of the molecular mechanisms related to the roles of lncRNAs in response to LP stress. This study increases our knowledge of lncRNAs and provides new insights into the function of lncRNAs in LP stress.

\section{Results}

Identification and characterization of IncRNAs across two soybean genotypes under different $P$ levels

To identify LP-responsive lncRNAs in soybean roots, we constructed 12 cDNA libraries from soybean root samples from two genotypes with contrasting responsiveness to LP stress, namely, Bogao (BG, a LP-sensitive genotype) and Nannong 94156 (NN94156, a LP-tolerant 
genotype), after exposure to high/normal phosphorus (HP, $500 \mu \mathrm{M}$, control) and low phosphorus (LP, $5 \mu \mathrm{M}$ ) conditions [32]. Three biological replicates of each condition were used to minimize the individual variation. The libraries were sequenced using the Illumina HiSeq 4000 platform, and 125-bp paired-end reads were generated. Approximately 1,087 million raw sequencing reads were generated from all 12 libraries, and each sample contained reads ranging from 75.5 to 100.7 million in number. After discarding adaptor sequences and lowquality reads $(\mathrm{Q}$-value $\leq 20)$, more than $90 \%$ of the total reads were retained [33]. We mapped these clean reads to the soybean reference genome sequence (Wm82.a2.v1). In total, 4,166 novel lncRNAs were predicted using the coding-noncoding index (CNCI) [34] and coding potential calculator (CPC) [35] under all tested conditions (Table S1).

The classification of these lncRNAs showed that the majority $(2,865,68.77 \%)$ of the 4,166 lncRNAs were located in intergenic regions, and the remaining 1,301 $(31.23 \%)$ resided within genic regions and included 454 bidirectional lncRNAs, 498 antisense lncRNAs, 121 sense lncRNAs, and 228 others that were not classified into these types (Fig. 1a). The type of lncRNA might be related to its functions; for example, overexpressed LAIR (a lncRNA transcribed from the antisense of the neighboring gene $L R K$ cluster) regulates the expression of several $L R K$ genes and increases the grain yield in rice [36].

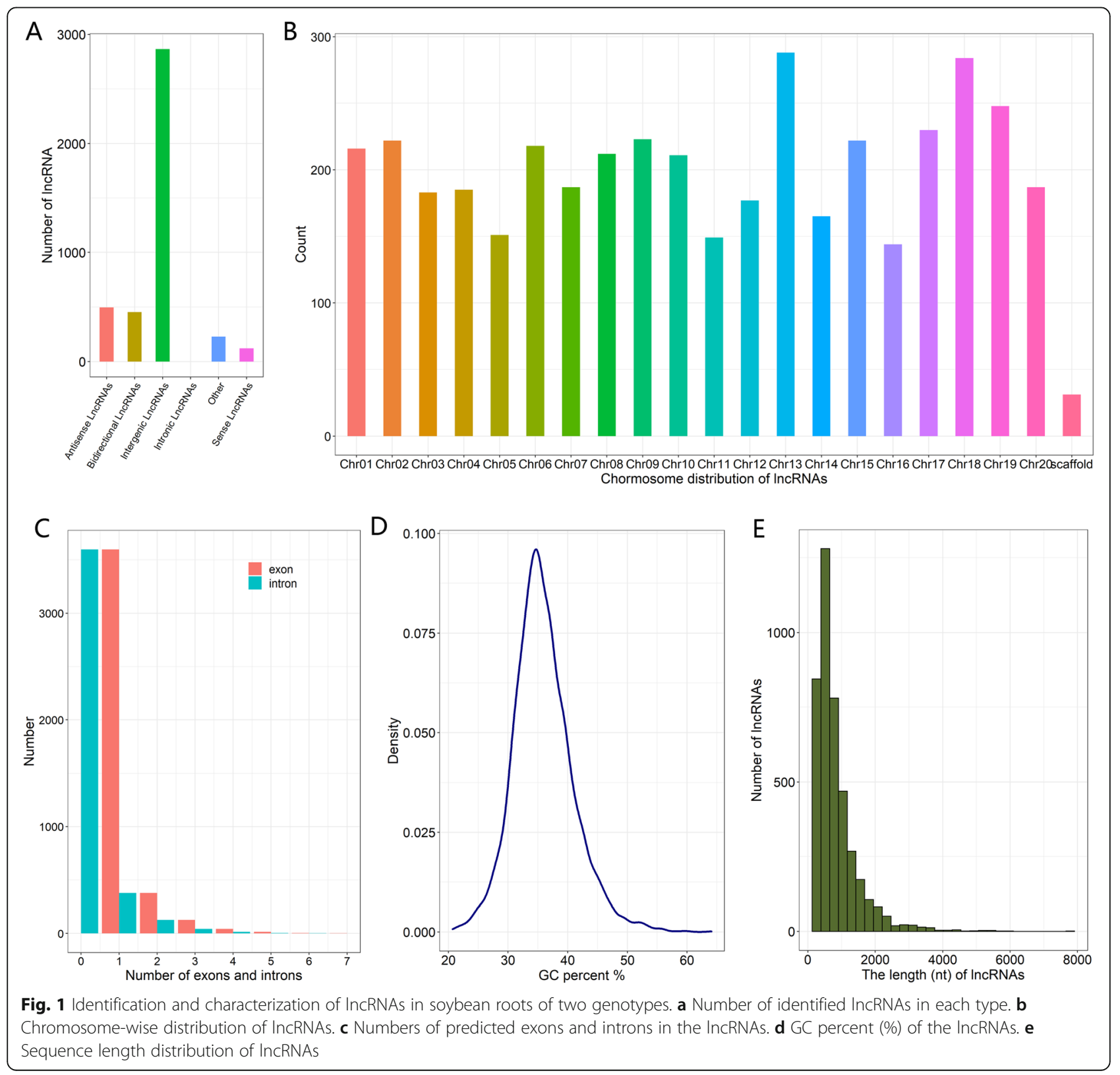



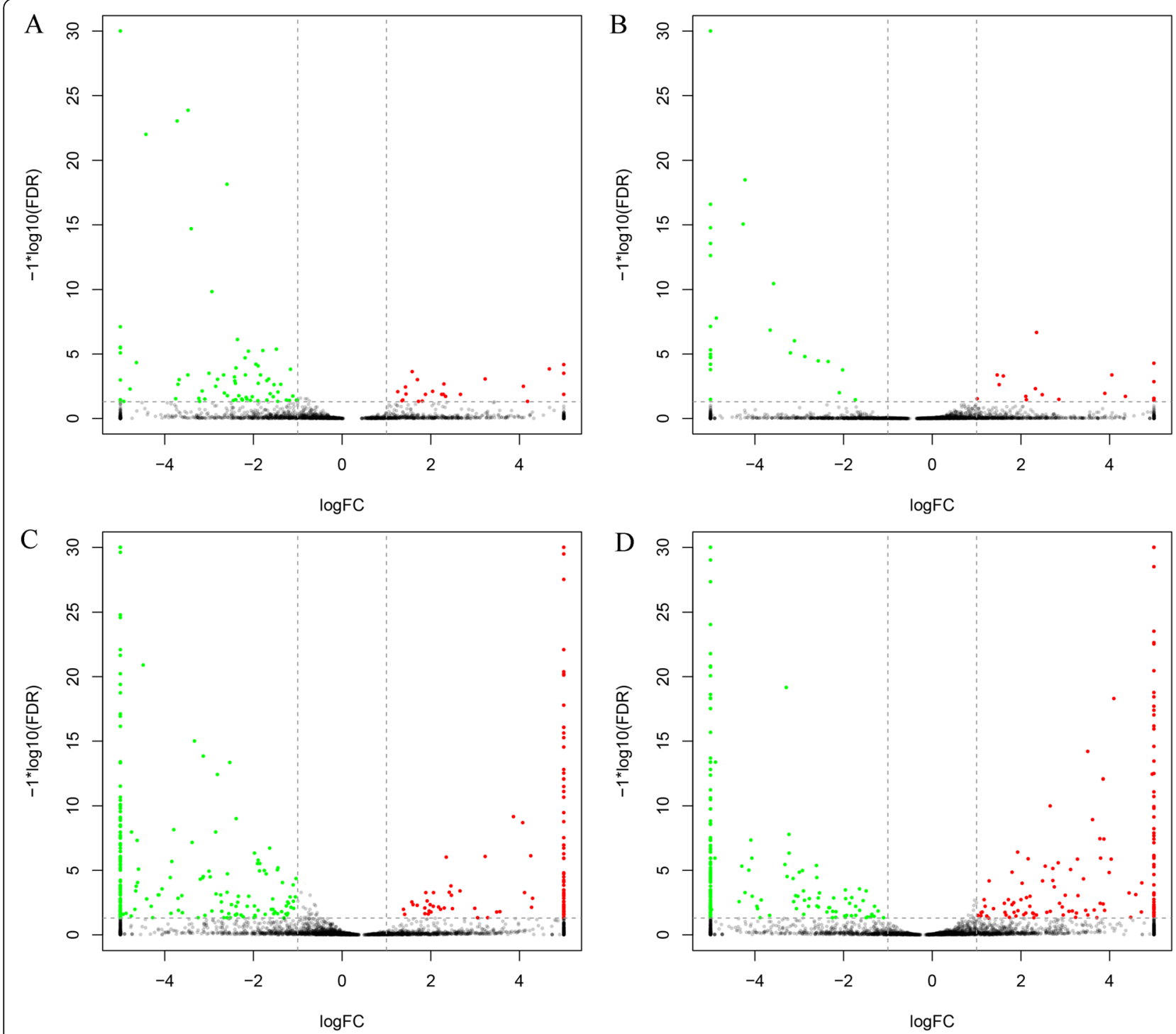

Fig. 2 Volcano plots of differentially expressed (DE) IncRNAs in soybean roots under different P conditions. a HP_BR vs. LP_BR. $\mathbf{b} H P \_N R$ vs. LP_NR. c HP_BR vs. HP_NR. $\mathbf{d}$ LP_BR vs. LP_NR. The red and green dots represent up- and downregulation, respectively. The $x$-axis represents the log2-fold change, and the $y$-axis represents the $\log _{10} p$-value. $P$-value $<0.05$ and $\mid \log _{2}$ fold change $>1$. HP and LP indicate high $P$ and low $P$, respectively, and BR and NR represent roots of Bogao and NN94156, respectively

We subsequently analyzed the chromosomal location of all the IncRNAs in the soybean genome. The distribution of the lncRNAs was uneven: chr13 and chr18 contained more than 250 lncRNAs, and chr05, chr11, and chr16 contained approximately 150 lncRNAs (Fig. 1b). In addition, we analyzed the number of exons and introns in each lncRNA transcript. Most of the lncRNAs contained one exon and no introns $(3,597)$, and the number of exons and introns was as high as seven and six, respectively (Fig. 1c). The GC content of the lncRNAs varied greatly, with a range of $20.68-64.1 \%$ and an average of $35.88 \%$.
The majority of lncRNAs have GC percent in the range of $30-45 \%$ (Fig. 1d). A majority $(94.43 \%)$ of the lncRNAs were shorter than 2,000 nucleotides (Fig. 1e).

\section{Differentially expressed (DE) IncRNAs in two soybean genotypes under different $P$ levels}

To identify the lncRNAs that are responsive to LP stress, we identified the differentially expressed (DE) transcripts of IncRNAs through pairwise comparisons between the two soybean genotypes under HP and LP conditions. The FPKM (fragments per kilobase of transcript per million mapped reads) values were used to evaluate the 


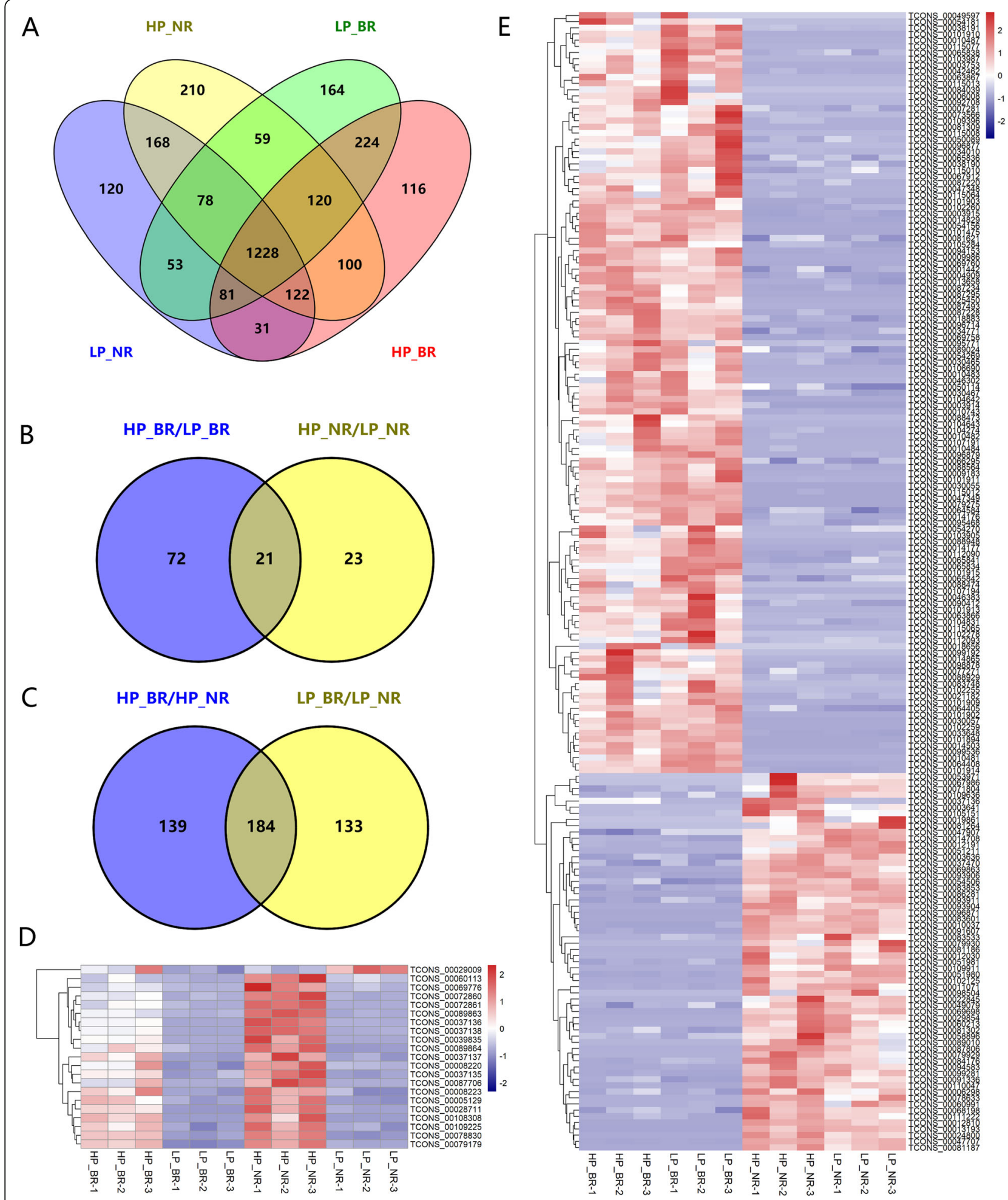

Fig. $3 \mathrm{DE}$ IncRNAs and expression patterns in soybean root plants under LP stress. a Venn diagram comparing the expressed IncRNAs in each root sample under different $\mathrm{P}$ levels. $\mathbf{b}$ Number of DE IncRNAs in the same genotype between different $\mathrm{P}$ levels. $\mathbf{c}$ Number of DE IncRNAs between different genotypes at the same P level. $\mathbf{d}$ Cluster analysis of the expression levels of common DE IncRNAs in the same genotype at different $\mathrm{P}$ levels. e Cluster analysis of the expression levels of common DE IncRNAs in different genotypes at the same P level 

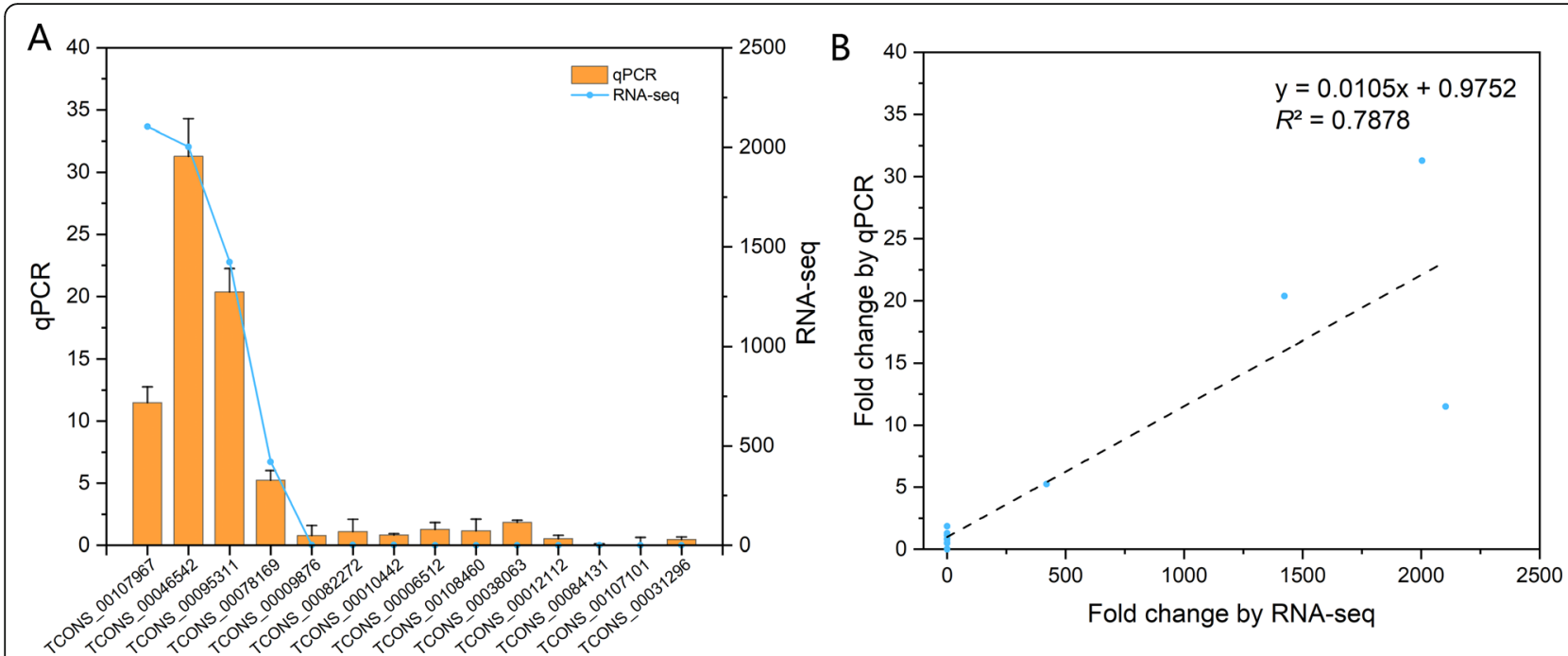

Fig. 4 Confirmation of the expression patterns of IncRNAs by qPCR. a Fold change obtained by IncRNA-seq and qPCR (LP/HP). b Linear regression analysis of IncRNA-seq and qPCR data

transcript abundance of lncRNAs. Differently expressed lncRNAs (referred to as DE lncRNAs hereafter) were defined as $\operatorname{lncRNAs}$ with $\log _{2} \mathrm{FC}>1$ and $\mathrm{FDR}<0.05$. In total, 525 DE lncRNAs were identified among the two different genotypes under HP and LP conditions, and these included 116 DE IncRNAs between different $P$ levels in the same genotype, 456 DE IncRNAs between different genotypes at the same P level, and 47 shared DE IncRNAs (Table S2). To identify the effect of LP stress on lncRNAs, we compared the DE lncRNAs of different genotypes under the same $\mathrm{P}$ condition and in the same genotype at different P levels (Fig. 2). As shown in the volcano plot, the LP treatment of Bogao and NN94156 resulted in more downregulated DE lncRNAs than upregulated DE lncRNAs, and the downregulated DE lncRNAs presented a more substantial change in differential expression than the upregulated DE IncRNAs (Fig. 2a and b). The number and fold change in expression of the upregulated and downregulated DE lncRNAs were relatively consistent in the Bogao and NN94156 genotypes under the same P level (Fig. 2c and d, Fig. S1).

Because the two genotypes showed markedly different responses to LP stress, we performed a Venn diagram analysis to elucidate the DE IncRNAs between the two genotypes under LP conditions. The number of common and unique DE lncRNAs between the two genotypes is indicated in the Venn diagram (Fig. 3a). NN94156 and Bogao shared 21 common DE lncRNAs in the HP vs. LP comparisons, and Bogao exhibited more genotypespecific DE lncRNAs (72) than NN94156 (23) (Fig. 3b), which is consistent with the results shown in the volcano plot (Fig. 2a and b). We found that the 21 common DE lncRNAs in Bogao were all downregulated under LP conditions, whereas most of these downregulated
lncRNAs (20, all except TCONS_00029009) were also downregulated in NN94156 (Fig. 3d). To determine whether the effect of LP stress on lncRNAs is related to genotype, we compared the changes in DE IncRNAs between Bogao and NN94156 under LP or HP conditions. The results identified 133 and 139 unique DE lncRNAs under the LP and HP conditions, respectively (Fig. 3c). The 184 common DE IncRNAs showed the same up- or downregulation trend: 123 were downregulated, and 61 were upregulated (Fig. 3e).

\section{Validation and quantification of IncRNAs}

To validate the expression of these LP-responsive lncRNAs, 14 lncRNAs were randomly selected and analyzed by quantitative PCR (qPCR). As shown in Fig. 4a, the expression patterns of the LP/HP IncRNAs determined by RNA-seq and qPCR were relatively consistent and presented similar trends. Both the qPCR and RNAseq assays revealed a positive correlation in the expression fold-change with an $R^{2}$ of 0.7878 (Fig. 4b), which indicated the robustness of our analysis and the reliability of the lncRNA expression patterns identified in the current study. These findings confirm that these lncRNAs are responsive to LP stress in soybean roots.

\section{Functions and expression patterns of DE IncRNAs and their target genes}

To reveal the potential functions of the differentially expressed lncRNAs under LP stress in two contrasting genotypes, we predicted the candidate targets of cis-, trans- and antisense-acting DE lncRNAs. In total, 785 targets of 374 DE lncRNAs were identified, and for 960 pairs, one lncRNA might have several targets and/or one mRNA target might be targets of several lncRNAs 

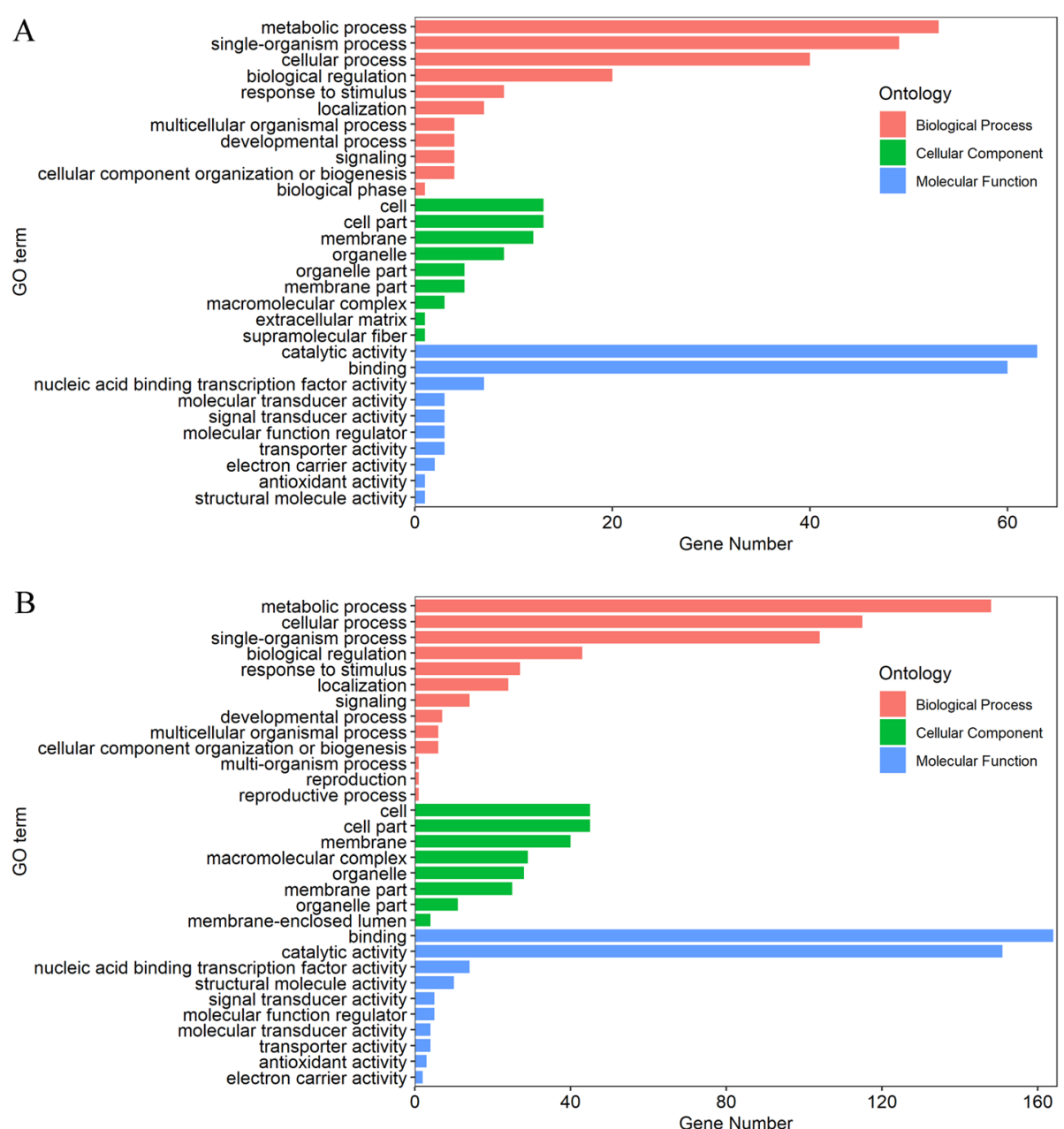

Fig. 5 Gene ontology (GO) enrichment of DE IncRNAs targets. a Targets of DE IncRNAs in the same genotype between different P levels. $\mathbf{b}$ Targets of DE IncRNAs between different genotypes at the same P level

(Table S3). To explore the putative functions of DE lncRNAs, we analyzed the Gene Ontology (GO) terms (Table S4) and Kyoto Encyclopedia of Genes and Genomes (KEGG) pathways of the putative target genes (Table S5).

The GO analysis of DE lncRNAs in one genotype at different P levels revealed that 403 GO terms (195 in the biological process category, 146 in the molecular function category, and 62 in the cellular component category) were significantly enriched $(P<0.05)$ (Fig. 5a). The analysis of the DE lncRNAs in Bogao or NN94156 exposed to the same P level showed that 1,086 GO terms (497 in the biological process category, 362 in the molecular function category, and 227 in the cellular component category) were significantly enriched $(P<0.05)$ (Fig. $5 b)$. Although the numbers of GO terms in the two genotypes were different, their trends were relatively similar. In brief, the most significant GO terms related to biological process were metabolic process, single-organism process, and cellular process, and the analysis of molecular functions revealed that catalytic activity and binding were the important significantly enriched GO terms. In addition, cell, cell part, membrane and organelle were the most important significant terms belonging to the cellular component categories. Taken together, these results show that these IncRNAs might play roles in a variety of biological processes that are responsive to LP stress.

We subsequently analyzed the enrichment of the predicted target genes of DE lncRNAs in KEGG pathways (Table S5). The targets of DE lncRNAs in the same genotype between different $\mathrm{P}$ levels were enriched in 42 KEGG pathways, including several KEGG pathways related to carbohydrate metabolism, lipid metabolism, and amino acid metabolism (Fig. 6a). For example, propanoate metabolism, glycolysis/gluconeogenesis, pyruvate 


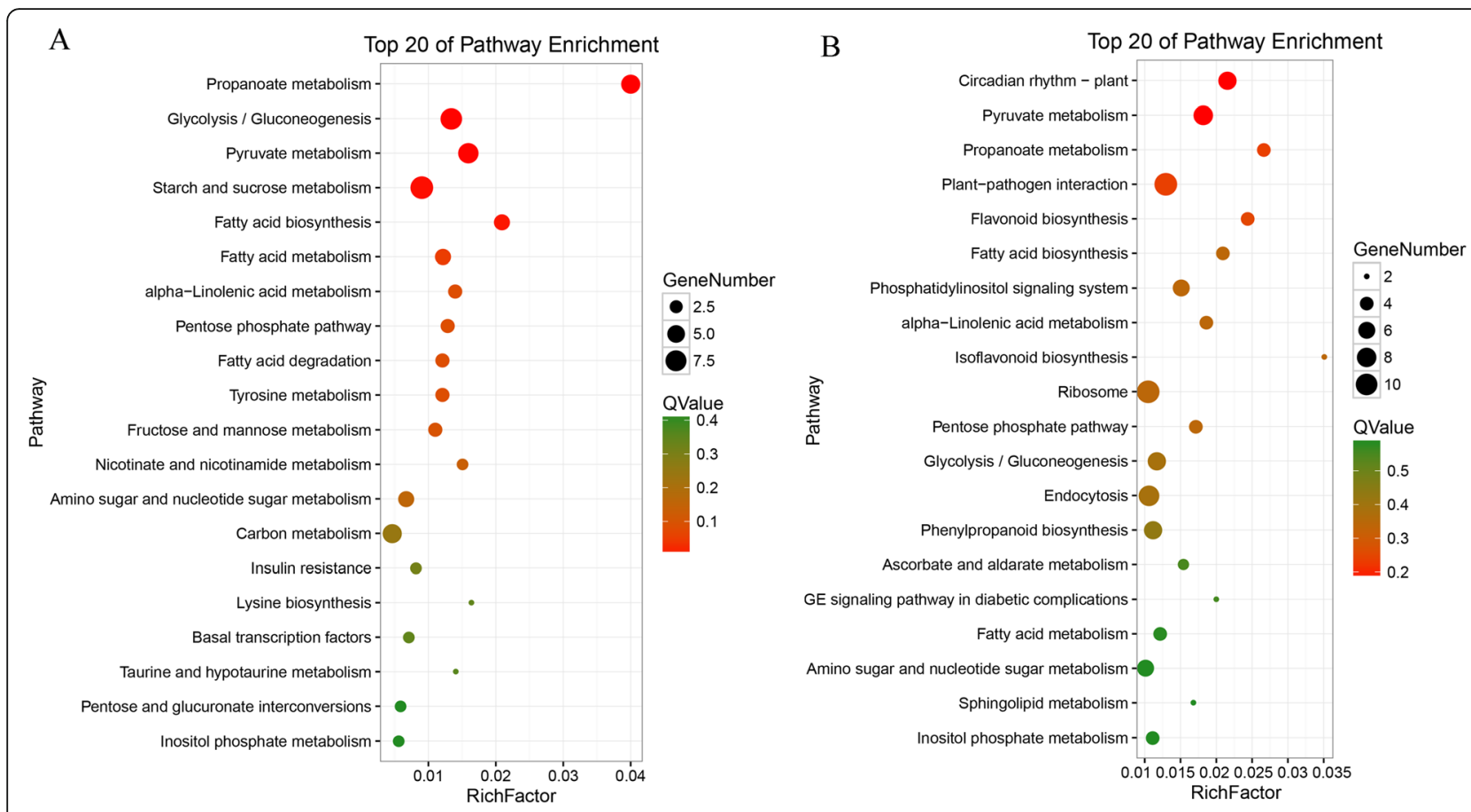

Fig. 6 KEGG enrichment of DE IncRNA targets. a Targets of DE IncRNAs in the same genotype between different P levels. b Targets of DE IncRNAs between different genotypes at the same P level

metabolism, starch and sucrose metabolism, and the pentose phosphate pathway belong to carbohydrate metabolism, and fatty acid biosynthesis, alpha-linolenic acid metabolism and fatty acid degradation are lipid metabolism pathways. The analysis of targets of DE lncRNAs between Bogao and NN94156 under the same conditions (HP and LP) showed that 74 KEGG terms were enriched, and these included environmental adaptation, carbohydrate metabolism, biosynthesis of other secondary metabolites, lipid metabolism, and signal transduction. Among the top 20 enriched pathways (Fig. 6b), circadian rhythm-plant and plant-pathogen interactions belong to environmental adaptation and were significantly enriched (Q-values $<0.05)$. Terms related to three secondary metabolite pathways, including flavonoid biosynthesis, isoflavonoid biosynthesis, and phenylpropanoid biosynthesis, were enriched. These findings suggest that DE lncRNAs might regulate genes involved in many biological processes, including molecular metabolism, energy synthesis and signal transduction, in response to LP stress.

\section{Putative P-related IncRNAs based on miRNAs}

miRNAs are endogenous noncoding RNAs with a size of 20 to 24 nucleotides that are generated from a singlestranded RNA precursor with a hairpin secondary structure. LncRNAs can be spliced by miRNAs into multiple small RNAs, and as a result, the function of lncRNAs can be regulated by miRNAs via posttranscriptional regulation. For example, miR399 is the first miRNA that was found to be upregulated specifically by $\mathrm{P}$ deficiency and rapidly decreased after $\mathrm{P}$ readdition, and certain miRNA families are responsive to $\mathrm{P}$ deficiency in various species [37].

Because our research mainly focused on LP stress, various targets, including lncRNAs and mRNAs of Prelated miRNAs, such as miR399, miR827, miR395, miR319, miR156, miR159, miR166, miR169, miR398 and miR447 [27, 37, 38], were selected. The lncRNA TCONS_00090111 was identified as a target of five miRNAs, namely, gma-miR156aa, gma-miR156z, gmamiR159b-3p, gma-miR159c, and gma-miR159f-3p. Similarly, TCONS_00015352 was predicted as a target of miR447-y (Table 1). The P-related miRNA targets were then predicted, and we found that nine mRNAs were also targets of lncRNAs (Table S6). As shown in Fig. 7, Glyma.19G121000, Glyma.02G109500 and TCONS_ 00068024 (a novel identified mRNA) were targets of IncRNAs and several miRNAs. Glyma.06G290000 and

Table 1 LncRNAs identified as targets of P-related miRNAs

\begin{tabular}{ll}
\hline miRNA & Target IncRNA \\
\hline gma-miR156aa & TCONS_00090111 \\
gma-miR156z & TCONS_00090111 \\
gma-miR159b-3p & TCONS_00090111 \\
gma-miR159c & TCONS_00090111 \\
gma-miR159f-3p & TCONS_00090111 \\
\hline
\end{tabular}




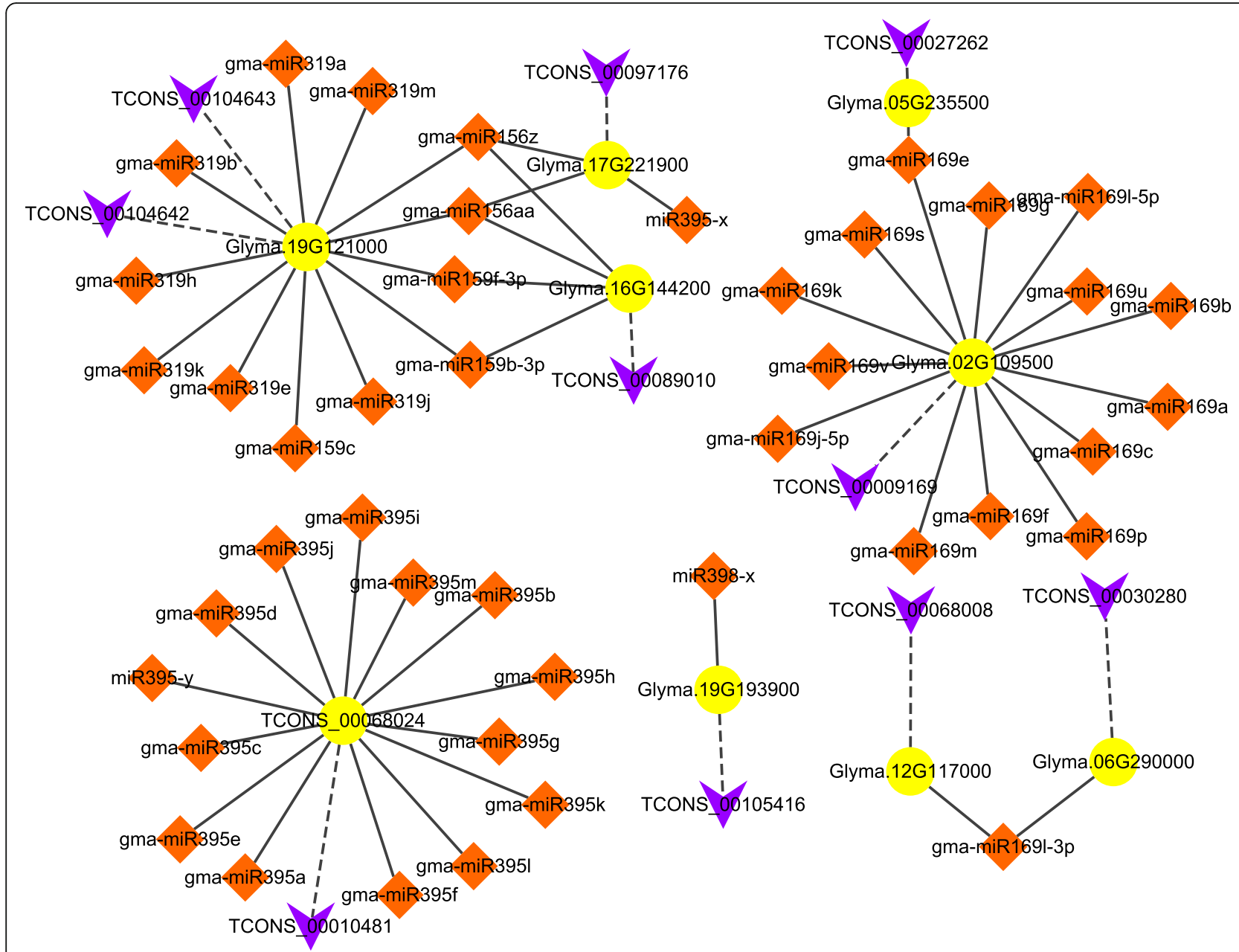

Fig. 7 Prediction of IncRNA-mRNA-miRNA networks associated with LP stress. The mRNA-IncRNA and miRNA-mRNA interaction networks were constructed based on P-related miRNAs. The yellow circles denote mRNAs, the orange rhombuses denote miRNAs, and the purple $V$ shapes indicate IncRNAs. The solid line represents a miRNA-mRNA interaction, and the dashed line indicates a IncRNA-mRNA interaction

Glyma.12G117000 were both annotated as ethyleneresponsive transcription factor 9-like mRNAs and were targets of the lncRNAs TCONS_00030280 and TCONS_ 00068008, respectively. Both genes were also targets of gma-miR169l-3p, which is a P-related miRNA [39]. Another example is Glyma.19G193900, which was predicted to be purple acid phosphatase 22-like, is the target of TCONS_00105416 and miR398-x, which belong to miR398 and have a demonstrated role in coping with P starvation stress [37].

Construction of the network of transcription factors (TFs) and P-related and plant hormone-associated IncRNAs and mRNAs

TFs regulate a diverse group of genes during stress responses and are important components of gene regulatory networks, and many TFs belonging to some families have been proven to play an important role in the maintenance of $\mathrm{P}$ homeostasis, such as the phosphate starvation response (PHR), bHLH, WRKY, ZAT, and MYB [40]. The P-mediated regulation of the root system architecture is driven by the local perception of $\mathrm{PO}_{4}{ }^{-}$at the root tip and involves changes in multiple plant hormones, such as auxin, gibberellins and ethylene, as well as hormonal changes coordinated with the root developmental responses to $\mathrm{P}$ availability [38]. P-related genes such as $\mathrm{PHO} 2$ and PHR1 play important roles in the $\mathrm{P}$ starvation response. To further study the function of lncRNAs in the responses of soybean roots to LP stress, we constructed a lncRNA-mRNA network of mRNAs of interest (including transcription factors and P-related and plant hormone targets) and corresponding lncRNAs according to the GO, KEGG and functional annotations of the target genes (Table S7). As shown in Fig. 8, the lncRNA-mRNA network consisted of 52 lncRNAs and 109 targets in total. Twenty-three lncRNAs might be involved in the regulation of gene transcription because their target genes have transcription factor activity; in 


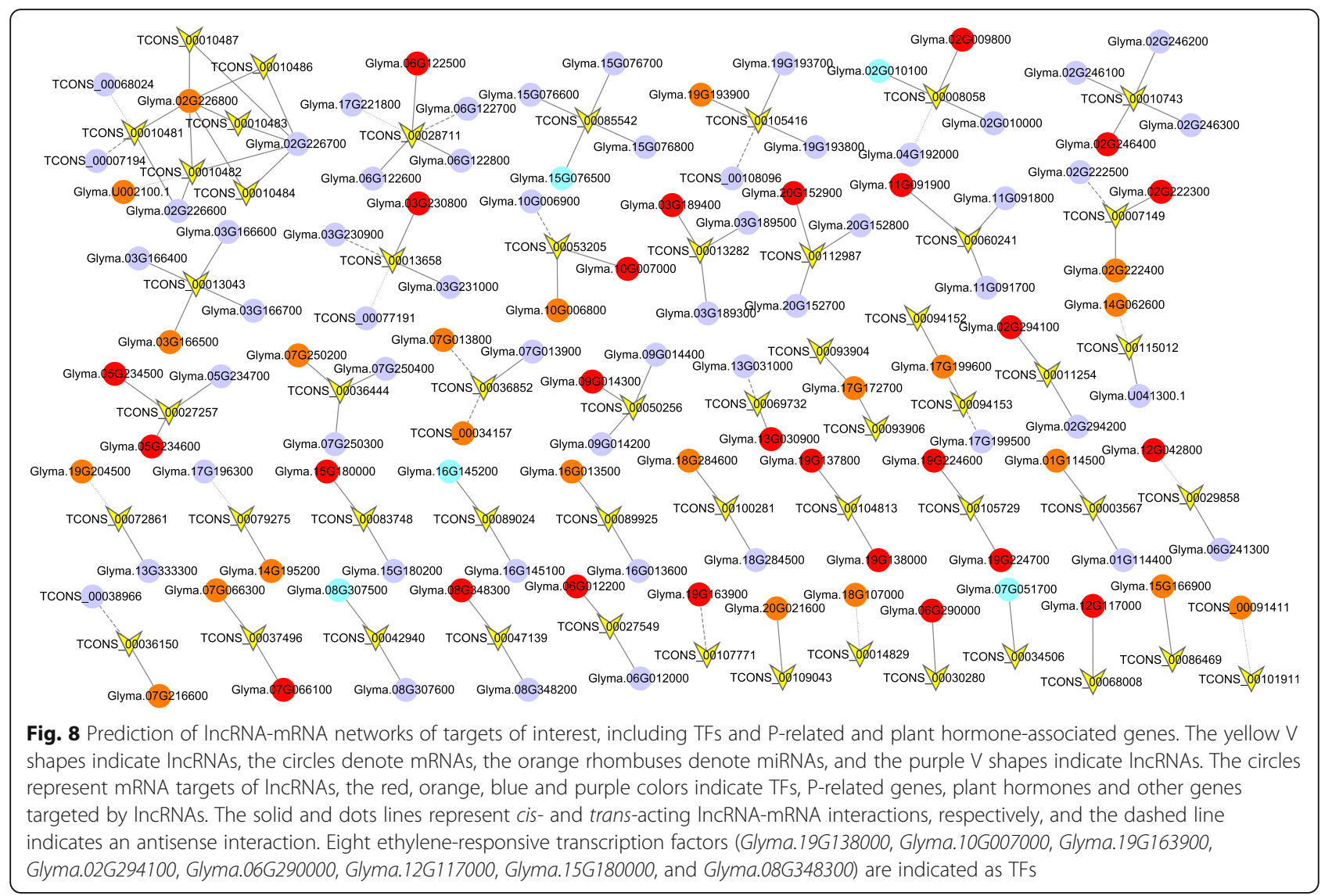

addition, three of the lncRNAs have two TF targets each, and 20 lncRNAs only have one TF target. The number of IncRNA targets varied from one to five, and Glyma.02G226800 and Glyma.02G226700 were targets of five and four lncRNAs, respectively. Twenty-six TFs belong to diverse families, such as MYB, bHLH, NAC, and AP2, and among these, MYB and bHLH reportedly play roles in the maintenance of $\mathrm{P}$ homeostasis [40]. Interestingly, we found that eight lncRNAs might be involved in ethylene regulation because their targets were annotated as ethylene-responsive TFs. Among the P-related genes, Glyma.17G172700 and Glyma.19G193900 were annotated as purple acid phosphatases (PAPs), and Glyma.20G021600 was predicted to be a phosphate transporter that is known as a PHT and is involved in LP stress.

\section{Discussion}

LncRNAs play important roles in a wide range of biological processes, particularly in plant reproductive development and responses to stresses [41]. However, little is known about their roles in LP stress, which is a major limiting factor in plant growth and the agricultural industry. Here, we undertook a genome-wide analysis of lncRNAs in two contrasting soybean genotypes subjected to phosphate starvation.

The number of lncRNAs varies greatly across plant species. For example, 48,345 lncRNAs have been identified in the maize transcriptome [15], and 1,212 novel IncRNAs have been found in Arabidopsis seedlings grown under $\mathrm{P}$-sufficient and $\mathrm{P}$-deficient conditions [14]. In this study, 4,166 lncRNAs were identified, and these lncRNAs show most of the common features of lncRNAs reported in other plants, such as short length, single exons, and low GC percent, which might be responsible for the common and ancient evolutionary origin of lncRNAs. In addition, we found that the sequence length of several (70) lncRNAs in soybean roots was longer than 3,000 bp. This finding is similar to the results from previous studies, which showed that 285 lncRNAs were longer than $3,000 \mathrm{bp}$ and that the length of 28 lncRNAs was longer than $10,000 \mathrm{bp}$ [42], and these results indicate the existence of a small number of long lncRNAs in plants. The type of lncRNAs was also highly variable in plants. We identified more intergenic lncRNAs $(2,865,68.77 \%)$ than other types, including antisense lncRNAs $(498,11.95 \%)$; in contrast, the number of antisense lncRNAs was greater than that of other types in Arabidopsis [14]. We questioned whether the 
number of exons was related to the length of the lncRNA gene, and our analysis revealed that the length of lncRNAs with a large number of exons was not significantly increased.

To identify the lncRNAs that were responsive to $\mathrm{P}$ stress, we identified the DE transcripts of the lncRNAs through pairwise comparisons between the two soybean genotypes under HP and LP conditions, and a total of 525 DE lncRNAs were identified among the two different genotypes under HP and LP conditions. As shown in Fig. 2a and $b$ and Fig. S1, the number of DE lncRNAs under LP stress identified in Bogao was greater than that found in NN94156, which indicated that Bogao is more sensitive to LP treatment, and this result is consistent with our previous findings that Bogao and NN94156 are P-sensitive and P-tolerant genotypes, respectively [30, 33]. Furthermore, our previous results revealed that all mRNAs, circular RNAs and lncRNAs in Bogao are more sensitive to LP stress than those of NN94156 [30, 33]. The two genotypes showed substantially different responses to LP stress; NN94156 and Bogao shared 21 common DE lncRNAs in the HP vs. LP comparisons, and Bogao exhibited more genotype-specific DE lncRNAs (72) than NN94156 (23) (Fig. 3b). Most of the common DE IncRNAs were constitutively downregulated in the two genotypes, which indicated that the biological mechanisms of lncRNAs involved in basal responsiveness to LP stress were conserved in both soybean genotypes. Although a similar trend in their response to LP stress was found for these shared lncRNAs, the degree of the change in expression was significantly different (Fig. 3d and e). To determine whether the effect of LP stress on lncRNAs is related to genotype, we compared the changes in DE lncRNAs between Bogao and NN94156 under LP or HP conditions. We identified 317 DE lncRNAs (181 downregulated, 136 upregulated) under LP conditions, which suggested that these lncRNAs were constitutively but differentially expressed between the two genotypes under LP conditions. The 133 DE lncRNAs unique to LP conditions might play a role in LP tolerance. In contrast, 139 lncRNAs were differentially expressed in the two genotypes only under HP conditions, which suggested that their differential expression is specific to LP stress.

Studies have shown that lncRNAs can directly bind to mRNAs by affecting the translation, shearing, and degradation of mRNAs and can also indirectly influence the expression of mRNAs [17]. Thus far, the mechanism underlying the interaction between lncRNAs and mRNAs has not been clarified. To reveal the potential functions of the DE IncRNAs under LP stress in the two contrasting genotypes, we predicted the candidate targets of the DE lncRNAs and then analyzed the GO terms and KEGG pathways of their putative target genes
(Table S5). The analysis of the DE lncRNAs in one genotype at different $\mathrm{P}$ levels and DE lncRNAs in Bogao or NN94156 exposed to the same P level revealed that 403 and 1,086 GO terms and 42 and 74 KEGG pathways were significantly enriched $(P<0.05)$, respectively (Fig. 5$)$. Our enrichment results showed that LP stress is a complex regulatory network involved in diverse biological processes, such as lipid metabolic processes, catalytic activity, cell membrane formation, signal transduction, and nitrogen fixation (Figs. 5 and 6), and these findings are supported by previous studies focusing on LP in soybean $[33,43]$. Previous research has shown that NtMYB12 acts as a phosphorus starvation response enhancement factor and regulates $N t C H S$ and NtPT2 expression, which results in increased flavonol and $\mathrm{P}$ accumulation and enhances tolerance to LP stress [44]. In this study, targets of lncRNAs were enriched in various KEGG pathways, including flavonoid, isoflavonoid and phenylpropanoid biosynthesis, which indicates that these lncRNAs might be involved in the synthesis of secondary metabolites to regulate P-responsive genes, but this hypothesis needs further research.

LncRNAs can be spliced by miRNAs into multiple small RNAs, and as a result, the function of lncRNAs can be regulated by miRNAs via posttranscriptional regulation [37]. The targets of 10 P-related miRNAs (miR399, miR827, miR395, miR319, miR156, miR159, miR166, miR169, miR398 and miR447) were predicted, and nine of them were also targets of lncRNAs (Table S6). Two lncRNAs, TCONS_00030280 and TCONS 00068008, exhibited shared mRNA targets (Glyma.06G290000 and Glyma.12G117000, annotated as ethylene-responsive transcription factor 9-like) with the P-related miR169 $l$-3p (Fig. 7). This finding was further supported by our recent study, which showed that NN94156 has the ability to tolerate LP stress via ethylene regulator-mediated enhanced $\mathrm{P}$ uptake and use efficiency in roots [32]. Therefore, lncRNAs might be partly involved in ethylene-mediated LP stress tolerance, and both genes are candidate genes that merit further investigation to gain further understanding of the involvement of lncRNAs in LP stress tolerance. Glyma.19G193900, which was predicted to be purple acid phosphatase 22-like, is the target of TCONS_00105416 and miR398- $x$, which belong to miR398 and have a demonstrated role in coping with $\mathrm{P}$ starvation stress [37]. PAPs are widely recognized as an adaptation strategy used by plants in response to $P$ deficiency, and the secretion of PAPs plays important roles in P acquisition [45]. The identification of other genes using our preliminary scenario suggested that lncRNAs are involved in the response to LP stress through the manipulation of genes with a variety of functionalities, and many of these genes might also be cotargets of P-associated miRNAs. A 
detailed investigation of these genes might result in an increased understanding.

The enhancement of root hair production, which increases the root surface area for nutrient uptake, is a typical adaptive response of plants to phosphate starvation [46]. Ethylene plays an important role in root hair development induced by $\mathrm{P}$ starvation by controlling root hair elongation [38]. According to the GO, KEGG and functional annotations of target genes of DE lncRNAs, TFs and P-related and plant hormone targets were selected to construct a lncRNA-mRNA network (Fig. 8). Interestingly, we found that eight lncRNAs might be involved in ethylene regulation because their targets were annotated as ethylene-responsive TFs. Ethyleneresponsive TFs belong to the APETALA2 (AP2)/ethylene response factor (ERF) family, which exists widely in plants. In Arabidopsis, the AP2/ERF TF superfamily comprises 147 members, and AP2/ERF proteins are known to regulate the responses of plants to various biotic and abiotic stresses and developmental processes. RNA interference and overexpression of AtERFO70 (AT1G71130), an ethylene response factor, results in alterations in the morphophysiological traits of roots and changes in the expression of a number of $\mathrm{P}$ starvationresponsive genes, which suggests a potential role for this $\mathrm{TF}$ in the maintenance of $\mathrm{P}$ homeostasis [40]. In addition to ethylene, auxin, GA and salicylic acid might be involved in the response to LP stress in soybean. The induction and secretion of acid phosphatases (APases) is considered an important strategy for improving plant growth under conditions of low inorganic phosphate. PAPs are an important class of plant APases that can be secreted into the rhizosphere to utilize organic phosphorus for plant growth and development [47]. Among the P-related genes, Glyma.17G172700 and Glyma.19G193900 were annotated as PAPs. Glyma.20G021600 was predicted to be a phosphate transporter that is known as a PHT and has important roles in $\mathrm{P}$ acquisition, allocation, and signal transduction. We speculate that their corresponding lncRNAs might have similar functions and are involved in LP stress.

\section{Conclusions}

The main aim of this research was to identify the potential IncRNA-related responses to LP stress in soybean and the differences in IncRNA responses between genotypes with different $\mathrm{P}$ efficiencies. Our results identified a total of 4,166 lncRNAs, including 525 DE lncRNAs, using roots of two representative genotypes under HP and LP conditions. LP stress can alter the genome-wide expression levels of lncRNAs, particularly in the Psensitive genotype Bogao. These findings might provide a first look at the landscape of IncRNAs in soybean in response to LP stress. Moreover, we identified several promising lncRNAs that might have potential value for further analysis of the mechanism underlying the response of soybean to LP stress. Overall, this study enriches the knowledge concerning lncRNAs and provides some clues for exploring the function of lncRNAs in the response of soybean to LP stress.

\section{Methods}

\section{Plant materials and growth conditions}

The LP-tolerant genotype Nannong 94156 (NN94156) and the LP-sensitive genotype Bogao were provided by Soybean Research Institute, Nanjing Agricultural University, China, and grown hydroponically as described previously [43]. In brief, the seeds were surfacesterilized, germinated and grown in an artificial intelligence climate chamber at $28 / 20^{\circ} \mathrm{C}$ with a 10 -h light/14-h dark photoperiod. When the two cotyledons had fully expanded, the soybean seedlings were transplanted into modified half-strength Hoagland's nutrient solution ( $\mathrm{pH} 5.8,500 \mu \mathrm{M}, \mathrm{KH}_{2} \mathrm{PO}_{4}$, sufficient $\mathrm{P}, \mathrm{HP}$ ). Three days later, half of the seedlings were transferred to Hoagland's nutrient solution with a low amount of $\mathrm{P}$ (5 $\mu \mathrm{M}$ P, LP), and the other half of the seeds were maintained under P-deficient conditions. The soybean plants were placed in the hydroponics box using a completely randomized block design. The solution was replenished every $3 \mathrm{~d}$, and $10 \mathrm{~d}$ after the plants were transferred to the P-deficient conditions, three independent biological replicates of the roots of seedlings were collected (12 samples in total) and stored at $-80{ }^{\circ} \mathrm{C}$ for total RNA extraction.

\section{RNA extraction, library construction, and Illumina sequencing}

RNA extraction and purity, library construction, and Illumina sequencing were performed according to $\mathrm{Lv}$ et al. [33]. Briefly, after total RNA extraction, rRNAs were removed using an Epicenter Ribo-Zero rRNA Kit (Epicenter, USA, cat: MRZSR116), and $1 \mu \mathrm{g}$ of rRNAdepleted RNA per sample was used to generate sequencing libraries according to the manual provided by Gene Denovo Biotechnology Co. (Guangzhou, China). The qualified libraries were then constructed and sequenced using an Illumina HiSeq 4000 platform. The 12 gene expression libraries were named HP-NR-1, HP-NR-2, HPNR-3; LP-NR-1, LP-NR-2, LP-NR-3; HP-BR-1, HP-BR-2, HP-BR-3; LP-BR-1, LP-BR-2, and LP-BR-3. The lncRNAs, mRNAs, miRNAs and circRNAs were sequenced simultaneously using the same samples and corresponding published results [32, 33, 48].

\section{Identification of IncRNAs}

The raw data were preprocessed to filter out adapters, reads containing more than $10 \%$ unknown nucleotides 
and reads containing more than $50 \%$ low-quality (Qvalue $\leq 20$ ). The obtained clean reads were then aligned to the soybean reference genome Williams 82 Wm82.a2.v1 using the splice read aligner TopHat2 [49]. Transcripts longer than 200 bp with exon numbers greater than 1 were selected. Two software programs, CNCI (coding-noncoding index) [34] and CPC (coding potential calculator) [35], were used to assess the protein-coding potential of the transcripts using the default parameters. The intersection of both nonproteincoding potential results was selected as long noncoding RNAs (lncRNAs). The lncRNA differential expression analysis between two different groups was performed using DESeq [50] software. Transcripts with a false discovery rate (FDR) below 0.05 were considered $\mathrm{DE}$ genes. Venn diagrams were generated using Venny 2.1.0 (https://bioinfogp.cnb.csic.es/tools/venny/index.html).

\section{Quantitative PCR (qPCR) validation of IncRNAs}

To validate the expression data obtained by RNA-seq, 14 lncRNAs were selected randomly for quantitative PCR (qPCR) analysis. qPCR experiments were performed with an ABI 7500 system (Applied Biosystems, Foster City, CA, USA). Each PCR contained $10 \mu \mathrm{L}$ of qPCR SYBR MIX (Toyobo, USA), $50 \mathrm{ng}$ of cDNA and $0.5 \mu \mathrm{L}$ of $10 \mu \mathrm{mol} \mathrm{L} \mathrm{L}^{-1}$ gene-specific primers. The PCR amplification procedure was $95^{\circ} \mathrm{C}$ for 5 min followed by 40 cycles of $95{ }^{\circ} \mathrm{C}$ for $15 \mathrm{~s}$ and $60{ }^{\circ} \mathrm{C}$ for $60 \mathrm{~s}$. The tubulin (GenBank accession: AY907703) gene in soybean was used as an internal control, and samples in which the cDNA template was replaced by $d_{d d} \mathrm{H}_{2} \mathrm{O}$ were used as a negative control. This experiment was performed with three technical replicates and three biological replicates, and the relative expression of lncRNAs was analyzed using the $2^{-\Delta \Delta C T}$ method [51]. The genes and their primers are listed in Table S8.

\section{Target gene prediction and functional analysis}

We searched for coding genes $10 \mathrm{~kb}$ upstream and downstream of the identified lncRNAs and then predicted them as cis-acting lncRNAs targeting neighboring genes. Some antisense lncRNAs might regulate gene silencing, transcription and mRNA stability. RNAplex software [52] (https://www.tbi.univie.ac.at/RNA/ RNAplex.1.html) was used to predict the complementary correlation of antisense lncRNAs and mRNAs, and mRNAs were predicted as antisense genes of lncRNAs. Another function of lncRNAs is the transregulation of coexpressed genes not adjacent to lncRNAs. The expression-related correlation between $\operatorname{lncRNAs}$ and protein-coding genes was analyzed to identify trans-acting genes of lncRNAs. These lncRNA target genes were functionally annotated using the GO (http:// geneontology.org/) and KEGG (http://www.genome.jp/ $\mathrm{kegg} /$ ) databases.

\section{Analyses of IncRNAs and/or mRNAs with miRNAs}

To find potential miRNA precursors, lncRNAs were aligned to miRBase, and those with more than $90 \%$ similarity were selected. In addition, miRPare software, which is based on the SVM method, was also used for the prediction of miRNA precursors. The lncRNAmRNA-miRNA network analysis was conducted as follows. First, 10 P-related miRNAs (miR399, miR827, miR395, miR319, miR156, miR159, miR166, miR169, miR398 and miR447) were selected, and PatMatch software (v1.2) was used to predict the target genes of these miRNAs. Second, nine common target genes were found to exist among the lncRNA and miRNA targets. Third, the common target genes of lncRNAs and miRNAs were integrated to form a lncRNA-mRNA-miRNA network. The lncRNA-mRNA network analysis proceeded as follows. First, TFs and P-related and plant hormone-related mRNAs were selected as mRNAs of interest according to their GO, KEGG and functional annotations. Second, the lncRNAs corresponding to these mRNAs of interest were searched. Third, all targets of these lncRNAs were selected, including mRNAs of interest (TFs and Prelated and plant hormone-associated genes), and noninterested targets were classified as none. Finally, a lncRNA-mRNA-miRNA network was constructed using Cytoscape 3.8.0 [53] software.

\section{Abbreviations}

IncRNAs: Long non-coding RNAs; ORF: Open reading frame; APA: Acid phosphatase activity; FDR: False Discovery Rate; DE: Differentially expressed; RNA-seq: RNA sequencing; FPKM: Fragments Per Kilobase of transcript per Million mapped reads; RSA: Root system architecture; P: Phosphorus;

LP: Low-phosphorus; CNCl: Coding-non-coding index; CPC: Coding potential calculator; qPCR: Quantitative PCR; KEGG: Kyoto Encyclopedia of Genes and Genomes; GO: Gene Ontology; PAPs: Purple acid phosphatases; AP2/ ERF: Apetala2/Ethylene Response Factor; TFs: Transcription factors

\section{Supplementary Information}

The online version contains supplementary material available at https://doi. org/10.1186/s12864-021-07750-8.

Additional file 1: Figure S1. Number of up- and downregulated DE IncRNAs under LP and HP conditions in the two soybean genotypes.

Additional file 2: Table S1. Detailed information of identified IncRNAs in soybean roots.

Additional file 3: Table S2. DE IncRNAs in different genotypes and P levels.

Additional file 4: Table S3. The target mRNAs of DE IncRNA in two comparisons.

Additional file 5: Table S4. GO enrichment analysis of the targeted mRNAs of significantly DE IncRNAs in two comparisons.

Additional file 6: Table S5. KEGG pathway annotation of the predicted target mRNAs of DE IncRNAs in two comparisons.

Additional file 7: Table S6. The mRNAs as targets of miRNA and IncRNA. 
Additional file 8: Table S7. The interested IncRNA-mRNA prediction for network construction.

Additional file 9: Table S8. List of primers used for qPCR of IncRNAs.

\section{Acknowledgements}

The authors thank to lab members for assistance.

\section{Authors' contributions}

$\mathrm{DZ}$ and $\mathrm{ZH}$ conceived and designed the experiments. HX, YY and XZ conducted the experiment. JZ, HX and DZ performed data analysis. JZ, XZ and $\mathrm{HX}$ wrote the manuscript. All authors read and approved the manuscript.

\section{Funding}

This research was funded by the Ministry of Science and Technology of China (2016YFD0100500), the key scientific and technological project of Henan Province (192102110023), the Henan agricultural university science and technology innovation fund (KJCX2019C02), the Key Scientific Research Projects of Higher Education Institutions in Henan Province (15B210007, 20A210017), Scientific Research Foundation for High-level Talent from Henan Institute of Science and Technology (201010617004) and the Postgraduate Education Reform and Quality Improvement Project of Henan Province (Yu degree [2018] No. 23).

\section{Availability of data and materials}

The datasets generated and/or analyzed during the current study are available in the NCBI Sequence Read Archive (SRA) Database, accession number SRP233239 (https://www.ncbi.nlm.nih.gov/sra/?term=SRP233239).

\section{Declarations}

Ethics approval and consent to participate

Not applicable.

\section{Consent for publication}

Not applicable.

\section{Competing interests}

The authors declare that they have no competing interest.

\section{Received: 27 August 2020 Accepted: 27 May 2021}

Published online: 09 June 2021

\section{References}

1. Djebali S, Davis CA, Merkel A, Dobin A, Lassmann T, Mortazavi A, Tanzer A, Lagarde J, Lin W, Schlesinger F, et al. Landscape of transcription in human cells. Nature. 2012:489(7414):101-8.

2. Marchese FP, Raimondi I, Huarte M. The multidimensional mechanisms of long noncoding RNA function. Genome Biol 2017;18(1):206

3. Rinn JL, Chang HY. Genome Regulation by Long Noncoding RNAs. Annu Rev Biochem. 2012;81:145-66.

4. Mishra A, Bohra A. Non-coding RNAs and plant male sterility: current knowledge and future prospects. Plant Cell Rep. 2018:37(2):177-91.

5. Ariel F, Romero-Barrios N, Jegu T, Benhamed M, Crespi M. Battles and hijacks: noncoding transcription in plants. Trends Plant Sci. 2015;20(6): 362-71.

6. Moran VA, Perera RJ, Khalil AM. Emerging functional and mechanistic paradigms of mammalian long non-coding RNAs. Nucleic Acids Res. 2012; 40(14):6391-400

7. Liu ZW, Zhao N, Su YN, Chen SS, He XJ. Exogenously overexpressed intronic long noncoding RNAs activate host gene expression by affecting histone modification in Arabidopsis. Sci Rep. 2020;10(1):3094.

8. Wang T, Xing J, Liu Z, Zheng M, Yao Y, Hu Z, Peng H, Xin M, Zhou D, Ni Z. Histone acetyltransferase GCN5-mediated regulation of long non-coding RNA At4 contributes to phosphate starvation response in Arabidopsis. J Exp Bot. 2019;70(21):6337-48

9. Chen L, Shi S, Jiang N, Khanzada H, Wassan GM, Zhu C, Peng X, Xu J, Chen $Y, Y u Q$, et al. Genome-wide analysis of long non-coding RNAs affecting roots development at an early stage in the rice response to cadmium stress. BMC Genomics. 2018;19(1):460.
10. Li L, Eichten SR, Shimizu R, Petsch K, Yeh CT, Wu W, Chettoor AM, Givan SA, Cole RA, Fowler JE, et al. Genome-wide discovery and characterization of maize long non-coding RNAs. Genome Biol. 2014;15(2):R40.

11. Zhao T, Tao X, Feng S, Wang L, Hong H, Ma W, Shang G, Guo S, He Y, Zhou $B$, et al. LncRNAs in polyploid cotton interspecific hybrids are derived from transposon neofunctionalization. Genome Biol. 2018;19(1):195.

12. Zhao X, Li J, Lian B, Gu H, Li Y, Qi Y. Global identification of Arabidopsis IncRNAs reveals the regulation of MAF4 by a natural antisense RNA. Nat Commun. 2018;9(1):5056.

13. Wang Y, Fan X, Lin F, He G, Terzaghi W, Zhu D, Deng XW. Arabidopsis noncoding RNA mediates control of photomorphogenesis by red light. Proc Natl Acad Sci U S A. 2014;111(28):10359-64.

14. Yuan J, Zhang Y, Dong J, Sun Y, Lim BL, Liu D, Lu ZJ. Systematic characterization of novel IncRNAs responding to phosphate starvation in Arabidopsis thaliana. BMC Genomics. 2016:17:655.

15. Huanca-Mamani W, Arias-Carrasco R, Cardenas-Ninasivincha S, Rojas-Herrera M, Sepulveda-Hermosilla G, Caris-Maldonado JC, Bastias E, Maracaja-Coutinho V. Long Non-Coding RNAs Responsive to Salt and Boron Stress in the Hyper-Arid Lluteno Maize from Atacama Desert. Genes (Basel). 2018:9(3):170.

16. Wang HV, Chekanova JA. Long Noncoding RNAs in Plants. Adv Exp Med Biol. 2017;1008:133-54.

17. Chekanova JA. Long non-coding RNAs and their functions in plants. Curr Opin Plant Biol. 2015;27:207-16.

18. Ding J, Lu Q, Ouyang Y, Mao H, Zhang P, Yao J, Xu C, Li X, Xiao J, Zhang Q. A long noncoding RNA regulates photoperiod-sensitive male sterility, an essential component of hybrid rice. Proc Natl Acad Sci U S A. 2012;109(7):2654-9.

19. Hirayama T, Shinozaki K. Research on plant abiotic stress responses in the post-genome era: past, present and future. Plant J. 2010;61 (6):1041-52.

20. Puga MI, Rojas-Triana M, de Lorenzo L, Leyva A, Rubio V, Paz-Ares J. Novel signals in the regulation of Pi starvation responses in plants: facts and promises. Curr Opin Plant Biol. 2017;39:40-9.

21. Wang F, Deng M, Xu J, Zhu X, Mao C. Molecular mechanisms of phosphate transport and signaling in higher plants. Semin Cell Dev Biol. 2018;74:114-22.

22. Zhang D, Song $H$, Cheng $H$, Hao D, Wang $H$, Kan G, Jin H, Yu D. The acid phosphatase-encoding gene GmACP1 contributes to soybean tolerance to low-phosphorus stress. PLoS Genet. 2014;10(1):e1004061.

23. Cordell D, Drangert J-O, White S. The story of phosphorus: Global food security and food for thought. Glob Environ Change. 2009;19(2):292-305.

24. Cai Z, Cheng Y, Xian P, Ma Q, Wen K, Xia Q, Zhang G, Nian H. Acid phosphatase gene GmHAD1 linked to low phosphorus tolerance in soybean, through fine mapping. Theor Appl Genet. 2018;131(8):1715-28.

25. Rubio V, Linhares F, Solano R, Martin AC, Iglesias J, Leyva A, Paz-Ares J. A conserved MYB transcription factor involved in phosphate starvation signaling both in vascular plants and in unicellular algae. Genes Dev. 2001; 15(16):2122-33

26. Fujii H, Chiou TJ, Lin Sl, Aung K, Zhu JK. A miRNA involved in phosphatestarvation response in Arabidopsis. Curr Biol. 2005;15(22):2038-43.

27. Li Z, Zhang X, Liu X, Zhao Y, Wang B, Zhang J. miRNA alterations are important mechanism in maize adaptations to low-phosphate environments. Plant Sci. 2016;252:103-17.

28. Du Q, Wang K, Zou C, Xu C, Li WX. The PILNCR1-miR399 Regulatory Module Is Important for Low Phosphate Tolerance in Maize. Plant Physiol. 2018; 177(4):1743-53.

29. Hu B, Zhu C, Li F, Tang J, Wang Y, Lin A, Liu L, Che R, Chu C. LEAF TIP NECROSIS1 plays a pivotal role in the regulation of multiple phosphate starvation responses in rice. Plant Physiol. 2011;156(3):1101-15.

30. Li H, Yang Y, Zhang H, Chu S, Zhang X, Yin D, Yu D, Zhang D. A Genetic Relationship between Phosphorus Efficiency and Photosynthetic Traits in Soybean As Revealed by QTL Analysis Using a High-Density Genetic Map. Front Plant Sci. 2016;7:924.

31. XiHuan L, WenSuo C, CaiYing Z. Advances of soybean (Glycine max L.) phosphorus nutrition and high P-eicient germplasms screening in China. Soybean Science. 2011;30(02):322-7.

32. Zhang $H$, Yang $Y$, Sun $C$, Liu X, Lv L, hu Z, Yu D, Zhang D. Up-regulating GmETO1 improves phosphorus uptake and use efficiency by promoting root growth in soybean. Plant Cell Environ. 2020;43(9):2080-94.

33. Lv L, Yu K, Lü H, Zhang X, Liu X, Sun C, Xu H, Zhang J, He X, Zhang D. Transcriptome-wide identification of novel circular RNAs in soybean in response to low-phosphorus stress. PLoS One. 2020;15(1):e0227243. 
34. Sun L, Luo H, Bu D, Zhao G, Yu K, Zhang C, Liu Y, Chen R, Zhao Y. Utilizing sequence intrinsic composition to classify protein-coding and long noncoding transcripts. Nucleic Acids Res. 2013;41(17):e166.

35. Kong L, Zhang Y, Ye ZQ, Liu XQ, Zhao SQ, Wei L, Gao G. CPC: assess the protein-coding potential of transcripts using sequence features and support vector machine. Nucleic Acids Res. 2007:35(Web Server issue):W345-349.

36. Wang Y, Luo X, Sun F, Hu J, Zha X, Su W, Yang J. Overexpressing IncRNA LAIR increases grain yield and regulates neighbouring gene cluster expression in rice. Nature Communications. 2018;9(1):3516.

37. Kuo HF, Chiou TJ. The role of microRNAs in phosphorus deficiency signaling. Plant Physiol. 2011;156(3):1016-24.

38. Oldroyd GED, Leyser O. A plant's diet, surviving in a variable nutrient environment. Science. 2020;368(6486):aba0196

39. Ning L-H, Du W-k, Song H-N, Shao H-B, Qi W-C, Sheteiwy MSA, Yu D-y. Identification of responsive miRNAs involved in combination stresses of phosphate starvation and salt stress in soybean root. Environ Exp Bot. 2019; 167:103823.

40. Ramaiah M, Jain A, Raghothama KG. ETHYLENE RESPONSE FACTOR070 Regulates Root Development and Phosphate Starvation-Mediated Responses. Plant Physiol. 2014;164(3):1484.

41. Zhang YC, Chen YQ. Long noncoding RNAs: new regulators in plant development. Biochem Biophys Res Commun. 2013;436(2):111-4.

42. Xu W, Yang T, Wang B, Han B, Zhou H, Wang Y, Li DZ, Liu A. Differential expression networks and inheritance patterns of long non-coding RNAs in castor bean seeds. Plant J. 2018;95(2):324-40.

43. Zhang D, Zhang H, Chu S, Li H, Chi Y, Triebwasser-Freese D, Lv H, Yu D. Integrating QTL mapping and transcriptomics identifies candidate genes underlying QTLs associated with soybean tolerance to low-phosphorus stress. Plant Mol Biol. 2017;93(1):137-50

44. Song Z, Luo Y, Wang W, Fan N, Wang D, Yang C, Jia H. NtMYB12 Positively Regulates Flavonol Biosynthesis and Enhances Tolerance to Low Pi Stress in Nicotiana tabacum. Front Plant Sci. 2020;10:1683-3.

45. Li C, Li C, Zhang H, Liao H, Wang X. The purple acid phosphatase GmPAP21 enhances internal phosphorus utilization and possibly plays a role in symbiosis with rhizobia in soybean. Physiol Plant. 2017;159(2):215-27.

46. Song L, Yu H, Dong J, Che X, Jiao Y, Liu D. The Molecular Mechanism of Ethylene-Mediated Root Hair Development Induced by Phosphate Starvation. PLOS Genetics. 2016;12(7):e1006194.

47. Kong Y, Li X, Wang B, Li W, Du H, Zhang C. The Soybean Purple Acid Phosphatase GmPAP14 Predominantly Enhances External Phytate Utilization in Plants. Front Plant Sci. 2018;9(292):1-10.

48. Liu X, Chu S, Sun C, Xu H, Zhang J, Jiao Y, Zhang D. Genome-wide identification of low phosphorus responsive microRNAs in two soybean genotypes by high-throughput sequencing. Funct Integr Genomics. 2020; 20(6):825-38.

49. Kim D, Pertea G, Trapnell C, Pimentel H, Kelley R, Salzberg SL. TopHat2: accurate alignment of transcriptomes in the presence of insertions, deletions and gene fusions. Genome Biol. 2013;14(4):R36.

50. Love Ml, Huber W, Anders S. Moderated estimation of fold change and dispersion for RNA-seq data with DESeq2. Genome Biol. 2014;15(12):550.

51. Livak KJ, Schmittgen TD. Analysis of relative gene expression data using real-time quantitative PCR and the 2(-Delta Delta C(T)) Method. Methods. 2001;25(4):402-8

52. Tafer H, Hofacker IL. RNAplex: a fast tool for RNA-RNA interaction search. Bioinformatics. 2008;24(22):2657-63.

53. Shannon P, Markiel A, Ozier O, Baliga NS, Wang JT, Ramage D, Amin N, Schwikowski B, Ideker T. Cytoscape: a software environment for integrated models of biomolecular interaction networks. Genome Res. 2003;13(11): 2498-504

\section{Publisher's Note}

Springer Nature remains neutral with regard to jurisdictional claims in published maps and institutional affiliations.

\section{Ready to submit your research? Choose BMC and benefit from:}

- fast, convenient online submission

- thorough peer review by experienced researchers in your field

- rapid publication on acceptance

- support for research data, including large and complex data types

- gold Open Access which fosters wider collaboration and increased citations

- maximum visibility for your research: over $100 \mathrm{M}$ website views per year

At BMC, research is always in progress.

Learn more biomedcentral.com/submissions 